\title{
FLORÍSTICA E ESTRUTURA DE UM REFLORESTAMENTO NO MACIÇO DA TIJUCA, RIO DE JANEIRO, RJ, BRASIL
}

\section{FLORISTIC AND STRUCTURE OF A REFORESTATION IN TIJUCA MASSIVE, RIO DE JANEIRO, RJ, BRAZIL}

\author{
Lucas Geromel de Góes \\ Programa de Engenharia Ambiental - PEA/UFRJ \\ Maria Fernanda Quintela \\ Laboratório de ecologia aplicada/UFRJ
}

\section{RESUMO}

O objetivo do presente trabalho foi avaliar a florística e estrutura de um reflorestamento com três anos e seis meses após o plantio, no Maciço da Tijuca, Rio de Janeiro, RJ, Brasil. Foram analisados todos os indivíduos do estrato arbustivo ou arbóreo, plantados e regenerantes, obtidos em amostragens realizadas em 44 parcelas de $25 \times 4 \mathrm{~m}$, instaladas aleatoriamente na área de reflorestamento. Foram amostrados 1550 indivíduos pertencentes a 95 espécies (42 plantadas, 25 exclusivas do estrato regenerante e 28 foram encontradas tanto plantadas como regenerantes), 74 gêneros e 34 famílias, para uma área total de 0,44 ha. $O$ dossel chegou aos sete metros, no entanto, a maioria dos indivíduos plantados ou regenerantes concentrou na classe de até $1 \mathrm{~m}$. A área basal foi estimada em $4,17 \mathrm{~m}^{2} / \mathrm{ha}$, sendo que as espécies pioneiras contribuíram com $79,4 \%$. A diversidade foi satisfatória devido a inclusão das espécies regenerantes no levantamento. A falta de manutenção afetou negativamente a estrutura do reflorestamento.

Palavras-chave: Avaliação, Monitoramento, Restauração florestal, Fitossociologia, Ecologia.

\begin{abstract}
The aim of this study was to evaluate the floristic and structure of a reforestation with three years and six months after planting, in the Massif of Tijuca, Rio de Janeiro, RJ, Brazil. We analyzed all individuals of tree communities, planted and regenerating obtained in samples collected in 44 plots of $25 \times 4 \mathrm{~m}$ randomly installed in the reforestation area. They sampled 1550 individuals belonging to 95 species (42 planted, 25 exclusive to the regenerative stratum and 28 were both found planted as regenerating), 74 genera and 34 families, a total area of 0.44 ha. The canopy arrived at seven meters, however, most individuals or regenerates planted in class concentrated to $1 \mathrm{~m}$. The basal area was estimated
\end{abstract}


at $4.17 \mathrm{~m}^{2} / \mathrm{ha}$, and the pioneer species contributed $79.4 \%$. Diversity was satisfactory due to inclusion of regenerating species in the survey. The lack of maintenance negatively affected the forestry structure.

Keywords: Evaluation, Monitoring, Forest restoration, Phytosociology, Ecology.

\section{INTRODUÇÃO}

O bioma da Mata Atlântica é uma das florestas mais diversas do mundo (DEAN, 2007). Apesar de tanta diversidade, a Mata Atlântica vem sendo degradada vorazmente, sendo considerado, um dos 25 hotspots de biodiversidade, áreas prioritárias para a conservação, que juntos possuem $44 \%$ das espécies de plantas vasculares e $35 \%$ das espécies de vertebrados, numa área que chega a apenas $1,4 \%$ da superfície terrestre (MYERS et al., 2000).

O município do Rio de Janeiro se encontra em $13^{\circ}$ lugar frente aos municípios com maior porcentual de Floresta Ombrófila do Estado, considerando um universo de 91 municípios (SOS MATA ATLÂNTICA \& INPE, 2009). Nesse sentido a prefeitura do Rio de Janeiro vem atuando, desde 0 início da década de 90 na recuperação de áreas degradadas de Mata Atlântica na cidade do Rio de Janeiro.

As pesquisas são a base para a compreensão e tentativa de reversão desse quadro de degradação. Além disso, muitas normas legais norteiam e orientam as etapas de recuperação de áreas degradadas, tanto no que tange as etapas iniciais quanto as finais, relacionadas a avaliações e monitoramentos dessas áreas.

A Resolução INEA № 89/2014, que atualiza a 36/2011 traz novas diretrizes para recuperação, recomposição, reabilitação ou restauração ecológica em áreas degradadas, no estado do Rio de Janeiro. Dentre as atualizações o Art. 8 enfatiza a importância da avaliação e monitoramento de áreas em processo de recuperação.

O objetivo deste trabalho foi avaliar e analisar a estrutura e composição florística dos indivíduos plantados e regenerantes em três áreas obtidas a partir de uma área em processo de recuperação, localizado no Maciço da Tijuca, zona oeste da cidade do Rio de Janeiro, RJ. 


\section{MATERIAL E MÉTODOS}

O presente estudo foi desenvolvido no Maciço da Tijuca, zona oeste da cidade do Rio de Janeiro, RJ. O reflorestamento estudado é parte do projeto de recuperação ambiental da bacia de Jacarepaguá, realizado em Julho de 2011, pela secretaria do meio ambiente da cidade do Rio de Janeiro (SMAC/CRA).

A metodologia utilizada nesse reflorestamento, foi através do plantio de espécies nativas, com espaçamento de $2 \times 2 \mathrm{~m}$, o que garantiu densidade de 2500 mudas por hectare. As mudas de 85 espécies foram plantadas em média com $60 \mathrm{~cm}$ de altura, em geral vigorosas.

A proporção das espécies plantadas foi de $10 \%$ de espécies da família Fabaceae (devido ao seu rápido crescimento); $65 \%$ de espécies nativas pioneiras; $15 \%$ de espécies nativas não pioneiras; e 10\% de espécies frutíferas nativas ou exóticas.

Para o plantio, não há informação sobre a proporção de indivíduos por espécie, porém, sabe-se que não ultrapassou $20 \%$ de indivíduos de uma mesma espécie. A distribuição das espécies, no momento do plantio, ocorreu ao acaso, sendo alternadas nas linhas espécies pioneiras e não pioneiras.

A vegetação original é floresta densa Montana que ocupava a faixa de $500 \mathrm{~m}$ a $1500 \mathrm{~m}$ de altitude, e Submontana nas faixas de $50 \mathrm{~m}$ a $500 \mathrm{~m}$.

O clima é do tipo tropical úmido, sem estação seca, megatérmico, do tipo Af, de acordo com o sistema de Köeppen. A precipitação média anual é de $1116 \mathrm{~mm}$ e o regime pluviométrico é definido sazonalmente com pequena deficiência hídrica entre os meses de julho e outubro.

A Cobertura vegetal do em torno é constituído por gramíneas, mas a matriz da paisagem é florestal, devido principalmente, ao Parque Nacional da Tijuca.

Para o levantamento florístico do presente estudo foram utilizadas 44 parcelas móveis de $25 \mathrm{~m} \times 4 \mathrm{~m}$, total de $100 \mathrm{~m}^{2}$ por parcela, ao todo foram amostrados 0,44 ha ou $1,7 \%$ da área total (25,73ha). 
Figura 1: Mapa de localização das áreas estudadas, bairro de Jacarepaguá, zona oeste da cidade do Rio de Janeiro, RJ, Brasil.

Estado do Rio de Janeiro

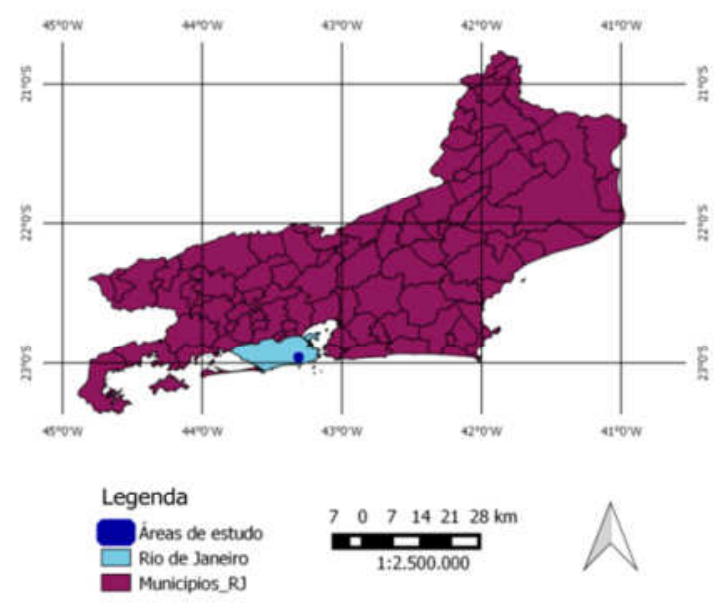

Cidade do Rio de Janeiro

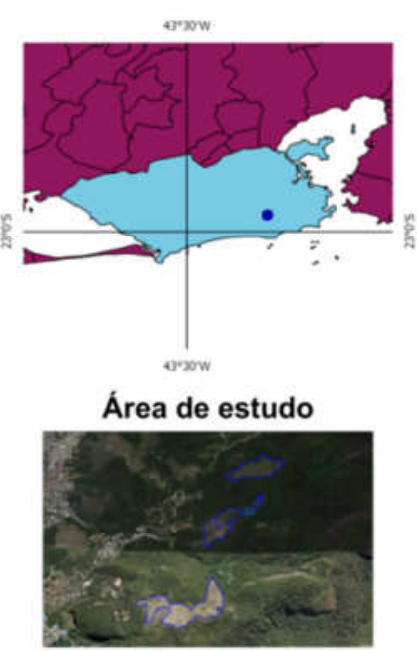

Em cada parcela foram realizadas as investigações, de modo a avaliar as espécies plantadas e as regenerantes, incluindo todos os indivíduos arbustivos - arbóreos encontrados dentro das parcelas.

As alturas foram obtidas com a utilização de barra graduada, e as medidas de circunferência, com a utilização de paquímetro ou fita métrica.

A identificação das espécies ocorreu na área ou coletadas, herborizadas e encaminhadas ao herbário da Universidade Federal do Rio de Janeiro, para a identificação através de especialistas, quando não foi possível a identificação no campo.

As espécies foram organizadas em listagem por famílias, de acordo com o Angiosperm Phylogeny Group (APG III, 2009). A nomenclatura foi atualizada segundo base de dados do site W3tropicos do Missouri Botanical Garden, disponível on-line (http://www.tropicos.org/).

Os grupos sucessionais e a classificação da síndrome de dispersão das espécies foram classificados em pioneiros e não pioneiros, segundo a lista indicada pela Resolução SMA $n^{0} 8 / 2008$, já que as legislações do estado do Rio de Janeiro não trazem essas informações detalhadas. As espécies não identificadas ou identificadas apenas em nível de gênero não foram classificadas. 
A estrutura da comunidade foi descrita a partir dos parâmetros fitossociológicos, tais como: Frequência Relativa (FrR), Abundância Relativa (AR), Dominância Relativa (DoR) e Valor de Importância (VI), segundo MUELLER-DOMBOIS \& ELLENBERG (1974).

A diversidade foi calculada pelo índice de Shannon $\left(\mathrm{H}^{\prime}\right)$, com base logarítmica natural, e também pelo índice de Pielou (J') (PIELOU, 1969).

\section{RESULTADOS E DISCUSSÃO}

O levantamento florístico na área amostral de 0,44ha diagnosticou a ocorrência de 1550 indivíduos arbustivos e arbóreos, pertencentes a 95 espécies, 74 gêneros e 37 famílias (Tabela 1). Entre essas espécies 83 são nativas regionais e 12 são exóticas. As famílias que apresentaram maior riqueza foram Fabaceae (25 espécies), Bignoniaceae (8 espécies), Myrtaceae (6 espécies), Malvaceae e Melastomataceae (5 espécies cada). As famílias com maior número de indivíduos foram Asteraceae $(n=624)$, Fabaceae $(n=358)$, Myrtaceae $(n=151)$, Bignoniaceae $(n=72)$, Melastomataceae $(n=57)$, Verbenaceae $(n=41)$ e Anacardiaceae $(n=32)$. A família que se distribuiu mais homogeneamente entre as áreas, com maior freqüência relativa foram Fabaceae, seguida de Bignoniaceae e Asteraceae.

Tabela 1. Relação de espécies e famílias encontradas em 44 parcelas de $25 \times 4 \mathrm{~m}$ no Maciço da Tijuca, Rio de Janeiro, RJ, Brasil com as respectivas informações de dispersão, grupo ecológico e demais informações. LEGENDA: D - Dispersão: Ane - Anemocórica; Zoo Zoocórica; Aut - Autocórica; GE - Grupo Ecológico: P - Pioneira; NP - Não Pioneira; E-exótica; NC- Não classificada; Origem: PL - Plantada; RG - Regenerante; ** Espécie não regional.

\begin{tabular}{llccc}
\hline Família & Nome científico & D & GE & Origem \\
\hline Anacardiaceae & Mangifera indica L** & Zoo & E & RG \\
Anacardiaceae & Myracrodruon urundeuva Allemão** & Aut & NP & PL \\
Anacardiaceae & Schinus terebinthifolius Raddi & Zoo & P & PL e RG \\
Annonaceae & Annona cacans Warm. & Zoo & P & RG \\
Apocynaceae & Tabernaemontana laeta Mart. & Zoo & P & RG \\
Arecaceae & Syagrus romanzoffiana Cham. & Zoo & NP & PL e RG \\
Asteraceae & Gochnatia polymorpha(Less.) Cabrera & Ane & P & RG \\
Bignoniaceae & Cybistax antisyphilitica (Mart.) Mart. & Ane & P & RG \\
Bignoniaceae & Handroanthus chrysotrichus (Mart. ex A. DC.) & Ane & P & PL e RG \\
Bignoniaceae & Handroanthus heptaphyllus (Vell.) Mattos & Ane & NP & PL \\
Bignoniaceae & Handroanthus impetiginosus Mattos** & Ane & NP & PL \\
Bignoniaceae & Handroanthus umbellatus (Sond.) Mattos & Ane & NP & PL \\
Bignoniaceae & Jacaranda mimosifolia D. Don ${ }^{* *}$ & Aut & E & PL \\
Bignoniaceae & Sparattosperma leucanthum (Vell.) K. Schum. & Ane & P & PL e RG
\end{tabular}




\begin{tabular}{|c|c|c|c|c|}
\hline Bignoniaceae & Zeyheria tuberculosa Bureau ex Verl. & Ane & NP & PL \\
\hline Boraginaceae & Cordia trichoclada DC. & Ane & $\mathrm{P}$ & PL e RG \\
\hline Boraginaceae & Cordia trichotoma (Vell.) Arráb. ex Steud. & Ane & $P$ & PL e RG \\
\hline Cannabaceae & Trema micrantha (L.) Blume & Zoo & $\mathrm{P}$ & $R G$ \\
\hline Clusiaceae & Garcinia gardneriana (Planch. \& Triana) Zappi & Zoo & NP & PL \\
\hline Cunoniaceae & Lamanonia cuneata (Cambess.) Kuntze $e^{* *}$ & Zoo & NP & $\mathrm{PL}$ \\
\hline Euphorbiaceae & Alchornea triplinervia (Spreng.) Müll. Arg. & Zoo & $P$ & PL e RG \\
\hline Euphorbiaceae & Croton urucurana Baill. & Aut & $\mathrm{P}$ & PL \\
\hline Euphorbiaceae & Joannesia princeps Vell. & Zoo & $P$ & PL \\
\hline Fabaceae & Anadenanthera colubrina (Vell.) Brenan & Aut & $\mathrm{P}$ & PL \\
\hline Fabaceae & Anadenanthera peregrina (L.) Speg. & Aut & $P$ & $P L$ e $R G$ \\
\hline Fabaceae & Apuleia leiocarpa (Vogel) J.F. Macbr. & Aut & NP & $R G$ \\
\hline Fabaceae & Bauhinia variegata $L .{ }^{* *}$ & Aut & $\mathrm{E}$ & $P L$ e RG \\
\hline Fabaceae & Caesalpinia peltophoroides Benth. & Aut & $P$ & PL \\
\hline Fabaceae & Dalbergia nigra (Vell.) Allemão ex Benth. & Aut & $P$ & $P L$ \\
\hline Fabaceae & Enterolobium contortisiliquum (Vell.) Morong & Zoo & $P$ & PL \\
\hline Fabaceae & Erythrina sp. & Aut & $\mathrm{P}$ & PL \\
\hline Fabaceae & Hymenaea courbaril L. & Zoo & NP & PL \\
\hline Fabaceae & Inga laurina (Sw.) Willd. & Zoo & $\mathrm{P}$ & PL \\
\hline Fabaceae & Inga vera Kunth & Zoo & $\mathrm{P}$ & PL \\
\hline Fabaceae & Libidibia ferrea (Mart.) L.P. Queiroz & Aut & NP & PL \\
\hline Fabaceae & Lonchocarpus muehlbergianus Hassl. & Aut & NP & PL e RG \\
\hline Fabaceae & Machaerium hirtum (Vell.) Stellfeld & Aut & NP & PL e RG \\
\hline Fabaceae & Machaerium nyctitans (Vell.) Benth. & Aut & NP & $R G$ \\
\hline Fabaceae & Mimosa bimucronata (DC.) Kuntze & Aut & $P$ & $R G$ \\
\hline \multicolumn{5}{|c|}{ Tabela1. (Continuação) } \\
\hline Família & Nome científico & D & GE & Origem \\
\hline Fabaceae & Peltophorum dubium (Spreng.) Taub. & Aut & $\mathrm{P}$ & PL e RG \\
\hline Fabaceae & Piptadenia gonoacantha (Mart.) J.F. Macbr. & Ane & $P$ & PL e RG \\
\hline Fabaceae & Piptadenia paniculata Benth. & Ane & $P$ & $R G$ \\
\hline Fabaceae & Pterocarpus rohrii Vahl & Ane & NP & PL e RG \\
\hline Fabaceae & Samanea saman (Jacq.) Merr. ${ }^{* *}$ & Aut & $P$ & PL \\
\hline Fabaceae & Schizolobium parahyba (Vell.) Blake & Aut & $P$ & $P L$ \\
\hline Fabaceae & Senna bicapsularis (L.) Roxb. & Aut & $\mathrm{P}$ & $P L$ \\
\hline Fabaceae & Senna multijuga (Rich.) H.S. Irwin \& Barneby & Aut & $P$ & $R G$ \\
\hline Fabaceae & Senna sp. & Aut & $\mathrm{P}$ & $R G$ \\
\hline Flacourtiaceae & Casearia sylvestris Sw. & Zoo & $P$ & $R G$ \\
\hline Indet. 1 & Indet. 1 & NC & NC & $R G$ \\
\hline Indet. 2 & Indet. 2 & $\mathrm{NC}$ & NC & PL \\
\hline Indet. 3 & Indet. 3 & NC & NC & PL \\
\hline Indet. 4 & Indet. 4 & NC & NC & $P L$ \\
\hline Indet. 5 & Indet. 5 & $\mathrm{NC}$ & NC & $R G$ \\
\hline Lamiaceae & Aegiphila sellowiana Cham. & Zoo & $P$ & PL e RG \\
\hline Lauraceae & Persea americana Mill. ${ }^{* *}$ & Zoo & $E$ & $R G$ \\
\hline Lecythidaceae & Cariniana ianeirensis $R$. Knuth & Ane & NP & $\mathrm{PL}$ \\
\hline Lecythidaceae & Lecythis pisonis Cambess. & Zoo & NP & $P L$ \\
\hline Lythraceae & Lafoensia glyptocarpa Koehne & Ane & NP & $\mathrm{PL}$ \\
\hline
\end{tabular}




\begin{tabular}{|c|c|c|c|c|}
\hline Lythraceae & Lafoensia pacari A.St.-Hil. & Ane & NP & PL \\
\hline Malvaceae & Bombacopsis glabra (Pasq.) Robyns & Zoo & NP & PL \\
\hline Malvaceae & Ceiba speciosa (A.St.-Hil.) Ravenna & Ane & NP & $\mathrm{PL}$ \\
\hline Malvaceae & Guazuma ulmifolia Lam. & Zoo & $P$ & PL \\
\hline Malvaceae & Luehea grandiflora Mart. \& Zucc. & Ane & NP & PL e RG \\
\hline Malvaceae & Pseudobombax grandiflorum (Cav.) A. Robyns & Ane & NP & PL \\
\hline Melastomataceae & Miconia Chartacea Triana & Zoo & $P$ & $R G$ \\
\hline Melastomataceae & Miconia sp. & Zoo & $P$ & PL e RG \\
\hline Melastomataceae & Miconia tristis Spring & Zoo & $P$ & $R G$ \\
\hline Melastomataceae & Tibouchina granulosa (Desr.) Cogn. & Ane & $P$ & $P L$ e $R G$ \\
\hline Melastomataceae & Tibouchina sellowiana (Cham.) Cogn. & Ane & $P$ & PL e RG \\
\hline Meliaceae & Cedrela fissilis Vell. & Aut & NP & PL \\
\hline Meliaceae & Cedrela sp. & Aut & NP & PL \\
\hline Meliaceae & Guarea guidonia (L.) Sleumer & Zoo & $P$ & PL e RG \\
\hline Moraceae & Brosimum glaziovii Taub. & Zoo & NP & $R G$ \\
\hline Moraceae & Ficus Iuschnathiana (Miq.) Miq. & Zoo & NP & $P L$ e $R G$ \\
\hline Moraceae & Ficus tomentella (Miq.) Miq. & Zoo & NP & PL \\
\hline Musaceae & Musa sp. ${ }^{* *}$ & Zoo & $E$ & $R G$ \\
\hline Myrtaceae & Campomanesia sp. & Zoo & NP & $R G$ \\
\hline Myrtaceae & Eugenia uniflora $L$. & Zoo & NP & $P L$ e $R G$ \\
\hline Myrtaceae & Psidium cattleianum Sabine & Zoo & NP & $R G$ \\
\hline Myrtaceae & Psidium guajava $L$. & Zoo & $P$ & PL e RG \\
\hline Myrtaceae & Psidium sp. & Zoo & NP & PL \\
\hline Myrtaceae & Syzygium cumini (L.) Skeels** & Zoo & $E$ & $R G$ \\
\hline Nyctaginaceae & Psonia sp. & Zoo & NP & PL \\
\hline Phytolaccaceae & Gallesia integrifolia (Spreng.) Harms & Ane & NP & PL \\
\hline \multicolumn{5}{|l|}{ Tabela1. (Continuação) } \\
\hline Família & Nome científico & D & GE & Origem \\
\hline Polygonaceae & Triplaris surinamensis Cham. ${ }^{* *}$ & Ane & $E$ & $\mathrm{PL}$ \\
\hline Rhamnaceae & Colubrina glandulosa Perkins & Zoo & NP & PL \\
\hline Rubiacea & Rudgea jasminoides (Cham.) Müll. Arg. & Zoo & NP & $R G$ \\
\hline Sapindaceae & Cupania oblongifolia Mart. & Zoo & $P$ & $R G$ \\
\hline Sapindaceae & Sapindus saponaria L. ${ }^{* *}$ & Zoo & NP & $P L$ e $R G$ \\
\hline Solanaceae & Solanum pseudoquina A. St.-Hil. & Zoo & $P$ & $P L$ e $R G$ \\
\hline Solanaceae & Solanum swartzianum Roem. \& Schult. & Zoo & $P$ & $P L$ e $R G$ \\
\hline Urticaceae & Cecropia hololeuca Miq. & Zoo & $P$ & PL e RG \\
\hline Urticaceae & Cecropia pachystachya Trécul & Zoo & $P$ & $P L$ e $R G$ \\
\hline Verbenaceae & Cytharexillum myrianthum Cham. & Zoo & $P$ & PL \\
\hline
\end{tabular}

Do total de espécies amostradas 28 foram encontradas tanto plantadas quanto regenerantes, 25 espécies apenas regenerantes e 42 espécies apenas plantadas.

$\mathrm{Na}$ classificação das espécies, de acordo com o grupo sucessional: $48,4 \%$ ou 46 espécies são pioneiras, 38,9\% ou 37 espécies são não pioneiras, 
7 espécies exóticas $(7,4 \%$ ) e 5 de classificação indefinida (5,3\%). Em relação aos indivíduos quanto ao grupo sucessional, foram encontrados os seguintes valores: $83 \%$ pioneiros, $15 \%$ não pioneiros, $1 \%$ exótico e $1 \%$ não classificados.

Para as espécies plantadas a porcentagem de não pioneiras é $44,3 \%$, as pioneiras $47,1 \%$. Já para as espécies regenerantes a porcentagem de espécies não pioneiras ficou muito abaixo com $25 \%$ e a grande maioria $(61,5 \%)$ foi pioneira (Figura 2).

Figura 2. Grupos sucessionais das espécies nas 44 parcelas amostrais do Maciço da Tijuca, Rio de Janeiro, RJ, Brasil. RG: Espécies Regenerantes; PL: Espécies Plantadas.

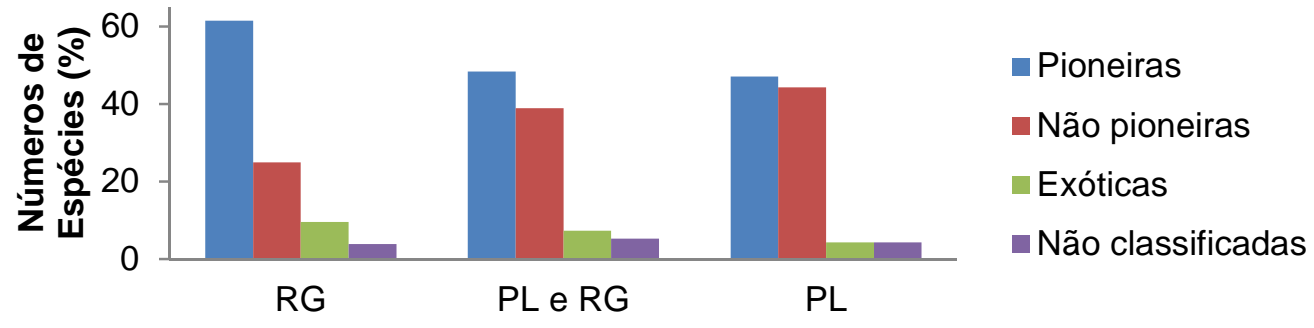

Com relação à síndrome de dispersão das espécies: 46,3\% são zoocóricas, 24,2\% são anemocóricas, 24,2\% são autocóricas e ainda 5,3\% não foram classificadas. Em relação à classificação dos indivíduos $27 \%$ são zoocóricos, $56 \%$ são anemocóricos, $17 \%$ são autocóricos. Para as espécies plantadas $41,4 \%$ são zoocóricas, $28,6 \%$ são anemocóricas, $25,7 \%$ são autocóricas e ainda 4,3\% não foram classificadas. Já para as espécies regenerantes $53,8 \%$ são zoocóricas, $23,1 \%$ são anemocóricas, $19,2 \%$ são autocóricas e ainda 3,8\% não foram classificadas.

As alturas dos indivíduos avaliados variaram de 0,1 a $12 \mathrm{~m}$, sendo 0,1 a $8 \mathrm{~m}$ para os indivíduos plantados e 0,1 a $12 \mathrm{~m}$ para os indivíduos regenerantes (Figura 3). A altura média foi $1,50 \mathrm{~m}$ e a mediana $1,0 \mathrm{~m}$. Os indivíduos plantados emergentes considerados maiores que 7,0m pertencem à espécie Tibouchina sellowiana. Já os indivíduos regenerantes emergentes considerados maiores que $12 \mathrm{~m}$ pertencem às espécies Alchornea triplinervea e Cecropia pachystachya.

Figura 3. Indivíduos arbustivos ou arbóreos amostrados nas 44 parcelas amostrais do Maciço da Tijuca, Rio de Janeiro, RJ, Brasil, distribuídos de acordo com a classe de altura (m), expressa em porcentagem. 


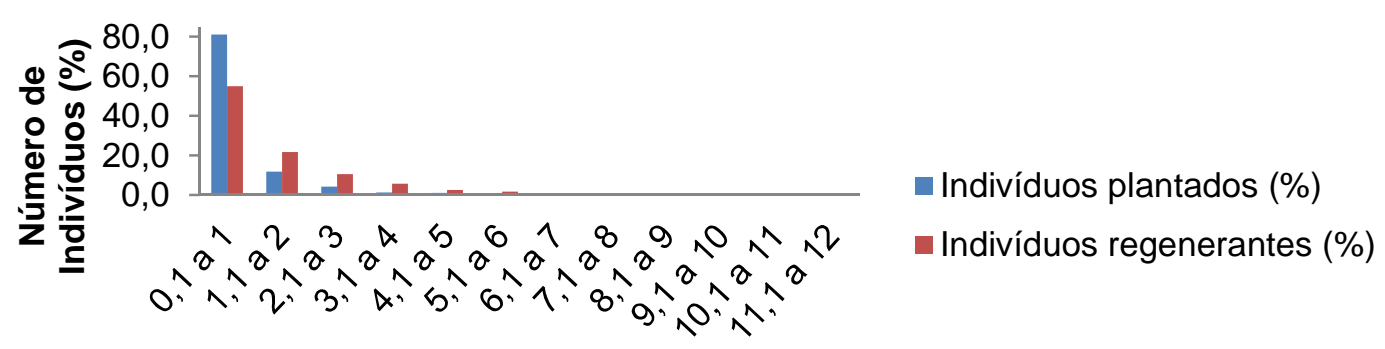

Classes de Altura (m)

Muitas das espécies plantadas ou regenerantes apresentaram alturas médias menores que $2 \mathrm{~m}$, do total de 95 espécies 73 mais especificamente, 0 que demonstra que a falta de manutenção influenciou negativamente 0 desenvolvimento horizontal das espécies.

A área basal dos indivíduos amostrados variou entre $0,00000079 \mathrm{~m}^{2}$ ou $0,007854 \mathrm{~cm}^{2}$ e $0,10892040 \mathrm{~m}^{2}$ ou $1089,20 \mathrm{~cm}^{2}$ (Figura 4). A média foi de $0,001186572 \mathrm{~m}^{2}$ e a mediana $0,000113097 \mathrm{~m}^{2}$. A área basal foi estimada em $4,179969 \mathrm{~m}^{2} / \mathrm{ha}$, para uma densidade estimada de 3522 indivíduos/ha. As espécies pioneiras contribuíram com $79,4 \%$, enquanto as espécies não pioneiras contribuíram com $20,6 \%$. As espécies plantadas ou regenerantes seguiram o mesmo padrão dos dois grupos juntos.

Já para as famílias os maiores valores de área basal foram encontrados em primeiro na Asteraceae e segundo Fabaceae se destacaram com 1,9005 $\mathrm{m}^{2} /$ ha e $0,7714 \mathrm{~m}^{2} /$ ha, respectivamente.

Figura 4. Indivíduos arbustivos ou arbóreos amostrados nas 44 parcelas amostrais do Macico da Tijuca, Rio de Janeiro, RJ, Brasil, distribuídos de acordo com a classe de área basal $\left(\mathrm{cm}^{2}\right)$, expressa em porcentagem. PL: Espécies Plantadas; RG: Espécies Regenerantes.

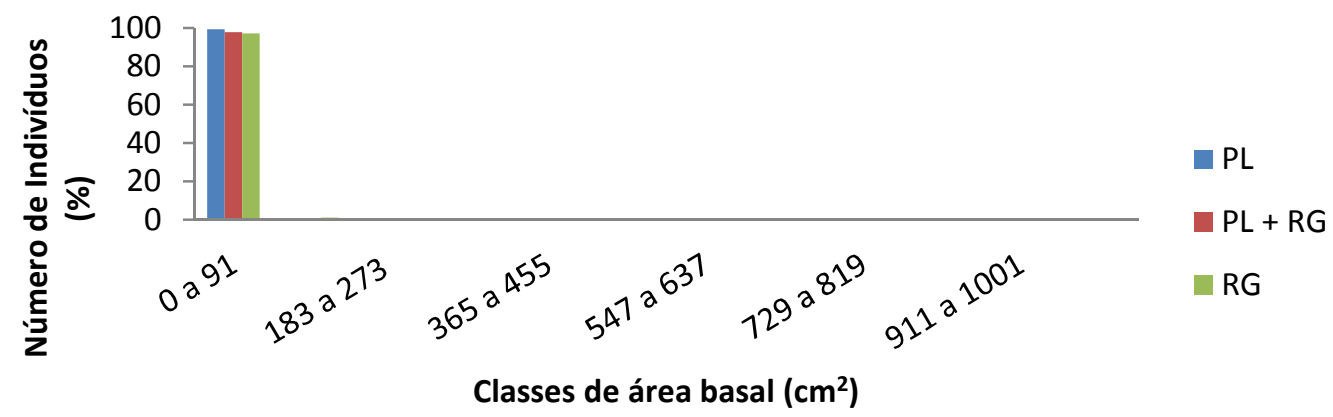

A espécie Gochnathia polymorpha apresentou, destacadamente, o maior valor de importância (VI) em relação às outras espécies, com base na análise dos parâmetros fitossociológicos (Tabela 2). Machaerium hirtum se destacou em segundo lugar. Psidium guajava e Peltophorum dubium apresentaram, respectivamente, terceiro e quarto maiores índices de importância, sendo os 
valores próximos para as duas espécies. As espécies que apresentaram um valor de VI entre 5 e 9 foram: Alchornea triplinervia, Pseudobombax grandiflorum, Sparattosperma leucanthum, Guarea guidonea, Cytharexillum myrianthum, Piptadenia gonoacantha, Inga laurina, Schinus terebinthifolius e Tibouchina sellowiana.

A diversidade encontrada no Maciço da Tijuca foi satisfatória, sendo o índice de Shannon $\left(\mathrm{H}^{\prime}\right)$ de 2,91. O índice de equitatividade de Pielou (J') foi 0,64 .

Tabela 2. Parâmetros fitossociológicos para as espécies do estrato arbustivas ou arbóreo, amostradas nas 40 parcelas amostrais do Maciço da Tijuca, Rio de Janeiro, RJ, Brasil. N: Número de indivíduos; A: Número de parcelas; DR: Densidade relativa; DoR: Dominância Relativa; FR: Frequência Relativa; VI: Valor de Importância; AB: Área Basal Média; Hm: Altura média; ${ }^{* \star}$ Espécies exóticas.

\begin{tabular}{|c|c|c|c|c|c|c|c|c|}
\hline Espécie & $\mathbf{N}$ & A & DR & DoR & FR & VI & AB & $\mathrm{Hm}$ \\
\hline Gochnatia polymorpha (Less.) Cabrera & 624 & 41 & 40,26 & 45,47 & 8,30 & 94,03 & 0,8362 & 1,57 \\
\hline Machaerium hirtum (Vell.) Stellfeld & 79 & 21 & 5,10 & 9,57 & 4,25 & 18,92 & 0,1760 & 2,31 \\
\hline Psidium guajava $L$. & 124 & 25 & 8,00 & 0,59 & 5,06 & 13,65 & 0,0108 & 0,70 \\
\hline Peltophorum dubium (Spreng.) Taub. & 50 & 24 & 3,23 & 3,69 & 4,86 & 11,77 & 0,0678 & 1,83 \\
\hline Alchornea triplinervia (Spreng.) Müll. Arg. & 15 & 11 & 0,97 & 6,05 & 2,23 & 9,25 & 0,1113 & 1,40 \\
\hline Pseudobombax grandiflorum (Cav.) A. Robyns & 15 & 9 & 0,97 & 4,96 & 1,82 & 7,75 & 0,0913 & 1,52 \\
\hline Sparattosperma leucanthum (Vell.) K. Schum. & 6 & 5 & 0,39 & 5,75 & 1,01 & 7,15 & 0,1058 & 3,83 \\
\hline Guarea guidonia (L.) Sleumer & 5 & 3 & 0,32 & 4,92 & 0,61 & 5,85 & 0,0905 & 2,44 \\
\hline Cytharexillum myrianthum Cham. & 41 & 11 & 2,65 & 0,55 & 2,23 & 5,42 & 0,0101 & 1,64 \\
\hline Piptadenia gonoacantha (Mart.) J.F. Macbr. & 36 & 12 & 2,32 & 0,59 & 2,43 & 5,34 & 0,0109 & 1,31 \\
\hline Inga laurina $(S w$.$) Willd.$ & 28 & 15 & 1,81 & 0,48 & 3,04 & 5,32 & 0,0088 & 1,71 \\
\hline Schinus terebinthifolius Raddi & 29 & 15 & 1,87 & 0,24 & 3,04 & 5,14 & 0,0043 & 0,95 \\
\hline Tibouchina sellowiana (Cham.) Cogn. & 31 & 10 & 2,00 & 1,11 & 2,02 & 5,13 & 0,0204 & 1,91 \\
\hline Inga vera Kunth & 19 & 12 & 1,23 & 1,31 & 2,43 & 4,97 & 0,0241 & 2,04 \\
\hline Mimosa bimucronata (DC.) Kuntze & 26 & 13 & 1,68 & 0,63 & 2,63 & 4,94 & 0,0116 & 1,44 \\
\hline Cybistax antisyphilitica (Mart.) Mart. & 24 & 14 & 1,55 & 0,48 & 2,83 & 4,86 & 0,0089 & 1,31 \\
\hline \multicolumn{9}{|l|}{ Tabela 2. (Continuação) } \\
\hline Espécie & $\mathbf{N}$ & A & DR & DoR & FR & VI & $A B$ & $\mathrm{Hm}$ \\
\hline Handroanthus chrysotrichus (Mart. ex A. DC. & 21 & 15 & 1,35 & 0,13 & 3,04 & 4,52 & 0,0024 & 1,11 \\
\hline Cecropia pachystachya Trécul & 6 & 6 & 0,39 & 2,87 & 1,21 & 4,47 & 0,0528 & 4,26 \\
\hline Lonchocarpus muehlbergianus Hassl. & 24 & 10 & 1,55 & 0,17 & 2,02 & 3,74 & 0,0031 & 0,83 \\
\hline Cordia trichotoma (Vell.) Arráb. ex Steud. & 24 & 7 & 1,55 & 0,63 & 1,42 & 3,59 & 0,0115 & 1,51 \\
\hline Anadenanthera peregrina (L.) Speg. & 14 & 11 & 0,90 & 0,12 & 2,23 & 3,25 & 0,0021 & 1,29 \\
\hline Persea americana Mill. & 2 & 2 & 0,13 & 2,71 & 0,40 & 3,25 & 0,0499 & 3,50 \\
\hline Solanum pseudoquina A. St.-Hil. & 24 & 5 & 1,55 & 0,50 & 1,01 & 3,06 & 0,0092 & 1,30 \\
\hline Cupania oblongifolia Mart. & 16 & 9 & 1,03 & 0,16 & 1,82 & 3,01 & 0,0029 & 1,54 \\
\hline Tibouchina granulosa (Desr.) Cogn. & 15 & 8 & 0,97 & 0,32 & 1,62 & 2,90 & 0,0058 & 1,78 \\
\hline Aegiphila sellowiana Cham. & 9 & 8 & 0,58 & 0,37 & 1,62 & 2,57 & 0,0068 & 1,58 \\
\hline Libidibia ferrea (Mart.) L.P. Queiroz & 9 & 8 & 0,58 & 0,07 & 1,62 & 2,27 & 0,0013 & 0,92 \\
\hline
\end{tabular}


Cariniana ianeirensis $R$. Knuth

Syzygium cumini (L.) Skeels

Enterolobium contortisiliquum (Vell.) Morong

Handroanthus heptaphyllus (Vell.) Mattos

Eugenia uniflora L.

Anadenanthera colubrina (Vell.) Brenan

Lafoensia glyptocarpa Koehne

Schizolobium parahyba (Vell.) Blake

Piptadenia paniculata Benth.

Senna bicapsularis (L.) Roxb.

Caesalpinia peltophoroides Benth.

Ceiba speciosa (A.St.-Hil.) Ravenna

Croton urucurana Baill.

Senna multijuga (Rich.) H.S. Irwin \& Barneby

Lafoensia pacari A.St.-Hil.

Samanea saman (Jacq.) Merr.

Psidium sp.

Gallesia integrifolia (Spreng.) Harms

Joannesia princeps Vell.

Bauhinia variegata $L$.

Miconia Chartacea Triana

Campomanesia sp.

Miconia sp.

Hymenaea courbaril L.

Solanum swartzianum Roem. \& Schult.

Luehea grandiflora Mart. \& Zucc.

Sapindus saponaria $L$.

Jacaranda mimosifolia D. Don

Cecropia hololeuca Miq.

Annona cacans Warm.

Handroanthus impetiginosus Mattos

Dalbergia nigra (Vell.) Allemão ex Benth.

Cordia trichoclada DC.

Guazuma ulmifolia Lam.

Casearia sylvestris $S w$.

Tabela 2. (Continuação)

\begin{tabular}{l} 
Espécie \\
\hline Indet. 5 \\
Triplaris surinamensis Cham. \\
Pterocarpus rohrii Vahl \\
Myracrodruon urundeuva Allemao \\
Garcinia gardneriana (Planch. \& Triana) Zappi \\
Ficus luschnathiana (Miq.) Miq. \\
Cedrela sp. \\
Handroanthus umbellatus (Sond.) Mattos \\
Musa sp. \\
Cedrela fissilis Vell.
\end{tabular}

\begin{tabular}{cccccccc}
8 & 7 & 0,52 & 0,17 & 1,42 & 2,10 & 0,0031 & 0,95 \\
1 & 1 & 0,06 & 1,83 & 0,20 & 2,09 & 0,0336 & 5,00 \\
7 & 5 & 0,45 & 0,47 & 1,01 & 1,94 & 0,0087 & 2,94 \\
8 & 6 & 0,52 & 0,07 & 1,21 & 1,81 & 0,0014 & 0,78 \\
11 & 5 & 0,71 & 0,01 & 1,01 & 1,73 & 0,0002 & 0,47 \\
8 & 5 & 0,52 & 0,15 & 1,01 & 1,68 & 0,0028 & 1,73 \\
9 & 5 & 0,58 & 0,07 & 1,01 & 1,66 & 0,0013 & 0,60 \\
6 & 5 & 0,39 & 0,22 & 1,01 & 1,62 & 0,0041 & 1,93 \\
7 & 5 & 0,45 & 0,07 & 1,01 & 1,54 & 0,0014 & 1,67 \\
7 & 5 & 0,45 & 0,03 & 1,01 & 1,50 & 0,0006 & 0,66 \\
5 & 5 & 0,32 & 0,11 & 1,01 & 1,45 & 0,0021 & 0,92 \\
8 & 4 & 0,52 & 0,09 & 0,81 & 1,42 & 0,0017 & 1,50 \\
5 & 5 & 0,32 & 0,07 & 1,01 & 1,40 & 0,0012 & 1,45 \\
6 & 4 & 0,39 & 0,06 & 0,81 & 1,26 & 0,0012 & 2,21 \\
6 & 4 & 0,39 & 0,06 & 0,81 & 1,25 & 0,0010 & 1,39 \\
7 & 2 & 0,45 & 0,37 & 0,40 & 1,23 & 0,0068 & 2,21 \\
6 & 4 & 0,39 & 0,02 & 0,81 & 1,22 & 0,0005 & 0,59 \\
5 & 4 & 0,32 & 0,09 & 0,81 & 1,22 & 0,0016 & 0,92 \\
5 & 4 & 0,32 & 0,07 & 0,81 & 1,20 & 0,0012 & 2,87 \\
6 & 3 & 0,39 & 0,01 & 0,61 & 1,00 & 0,0001 & 0,48 \\
5 & 3 & 0,32 & 0,03 & 0,61 & 0,96 & 0,0005 & 1,27 \\
8 & 2 & 0,52 & 0,03 & 0,40 & 0,95 & 0,0005 & 1,35 \\
5 & 3 & 0,32 & 0,02 & 0,61 & 0,95 & 0,0003 & 0,40 \\
4 & 3 & 0,26 & 0,05 & 0,61 & 0,92 & 0,0010 & 1,28 \\
3 & 3 & 0,19 & 0,01 & 0,61 & 0,81 & 0,0002 & 0,52 \\
3 & 3 & 0,19 & 0,01 & 0,61 & 0,81 & 0,0001 & 1,67 \\
3 & 3 & 0,19 & 0,01 & 0,61 & 0,81 & 0,0001 & 0,18 \\
5 & 2 & 0,32 & 0,02 & 0,40 & 0,75 & 0,0004 & 1,88 \\
4 & 2 & 0,26 & 0,09 & 0,40 & 0,75 & 0,0016 & 1,71 \\
3 & 2 & 0,19 & 0,13 & 0,40 & 0,73 & 0,0024 & 2,43 \\
4 & 2 & 0,26 & 0,03 & 0,40 & 0,69 & 0,0005 & 1,28 \\
2 & 2 & 0,13 & 0,15 & 0,40 & 0,69 & 0,0028 & 2,65 \\
2 & 2 & 0,13 & 0,13 & 0,40 & 0,67 & 0,0025 & 1,70 \\
2 & 2 & 0,13 & 0,13 & 0,40 & 0,66 & 0,0024 & 1,43 \\
3 & 2 & 0,19 & 0,05 & 0,40 & 0,65 & 0,0010 & 1,81 \\
\hline
\end{tabular}

\begin{tabular}{cccccccc} 
N & A & DR & DoR & FR & VI & AB & Hm \\
\hline 3 & 2 & 0,19 & 0,02 & 0,40 & 0,62 & 0,0005 & 0,95 \\
3 & 2 & 0,19 & 0,02 & 0,40 & 0,62 & 0,0004 & 0,50 \\
3 & 2 & 0,19 & 0,01 & 0,40 & 0,60 & 0,0001 & 0,63 \\
2 & 2 & 0,13 & 0,05 & 0,40 & 0,58 & 0,0009 & 1,48 \\
2 & 2 & 0,13 & 0,01 & 0,40 & 0,55 & 0,0002 & 1,75 \\
2 & 2 & 0,13 & 0,01 & 0,40 & 0,54 & 0,0002 & 0,49 \\
1 & 2 & 0,06 & 0,02 & 0,40 & 0,49 & 0,0004 & 0,75 \\
3 & 1 & 0,19 & 0,03 & 0,20 & 0,43 & 0,0006 & 0,81 \\
1 & 1 & 0,06 & 0,12 & 0,20 & 0,38 & 0,0021 & 2,10 \\
2 & 1 & 0,13 & 0,04 & 0,20 & 0,37 & 0,0007 & 0,43
\end{tabular}


Erythrina sp.

Lamanonia cuneata (Cambess.) Kuntze

Zeyheria tuberculosa Bureau ex Verl.

Senna sp.

Machaerium nyctitans (Vell.) Benth.

Brosimum glaziovii Taub.

Psonia sp.

Indet. 3

Psidium cattleianum Sabine

Tabernaemontana laeta Mart.

Trema micrantha (L.) Blume

Bombacopsis glabra (Pasq.) Robyns

Apuleia leiocarpa (Vogel) J.F. Macbr.

Lecythis pisonis Cambess.

Ficus tomentella (Miq.) Miq.

Colubrina glandulosa Perkins

Miconia tristis Spring

Syagrus romanzoffiana Cham.

Indet. 2

Rudgea jasminoides (Cham.) Müll. Arg.

Indet. 1

Indet. 4

Mangifera indica $L$. $\begin{array}{lll}2 & 1 & 0,13\end{array}$

210,13

110,06

110,06

110,06

110,06

110,06

1110,06

110,06

110,06

110,06

$\begin{array}{lll}1 & 1 & 0,06\end{array}$

$\begin{array}{lll}1 & 1 & 0,06\end{array}$

110,06

$\begin{array}{lll}1 & 1 & 0,06\end{array}$

110,06

110,06

110,06

110,06

110,06

110,06

110,06

110,06 $\begin{array}{lllll}0,01 & 0,20 & 0,34 & 0,0002 & 1,85\end{array}$

$\begin{array}{llllll}0,01 & 0,20 & 0,34 & 0,0001 & 0,63\end{array}$

$\begin{array}{llllll}0,05 & 0,20 & 0,32 & 0,0010 & 3,00\end{array}$

$\begin{array}{llllll}0,05 & 0,20 & 0,32 & 0,0009 & 3,00\end{array}$

$\begin{array}{llllll}0,03 & 0,20 & 0,30 & 0,0005 & 0,37\end{array}$

$\begin{array}{lllll}0,02 & 0,20 & 0,29 & 0,0005 & 3,00\end{array}$

$\begin{array}{llllll}0,02 & 0,20 & 0,29 & 0,0003 & 0,90\end{array}$

$\begin{array}{llllll}0,01 & 0,20 & 0,28 & 0,0003 & 0,73\end{array}$

$\begin{array}{llllll}0,01 & 0,20 & 0,28 & 0,0002 & 1,00\end{array}$

$\begin{array}{llllll}0,01 & 0,20 & 0,28 & 0,0002 & 1,05\end{array}$

$\begin{array}{llllll}0,01 & 0,20 & 0,28 & 0,0002 & 1,20\end{array}$

$\begin{array}{llllll}0,01 & 0,20 & 0,28 & 0,0002 & 0,80\end{array}$

$\begin{array}{llllll}0,01 & 0,20 & 0,28 & 0,0002 & 2,10\end{array}$

$\begin{array}{llllll}0,01 & 0,20 & 0,27 & 0,0001 & 2,31\end{array}$

$\begin{array}{llllll}0,01 & 0,20 & 0,27 & 0,0001 & 2,05\end{array}$

$\begin{array}{llllll}0,00 & 0,20 & 0,27 & 0,0001 & 1,21\end{array}$

$\begin{array}{llllll}0,00 & 0,20 & 0,27 & 0,0001 & 2,00\end{array}$

$\begin{array}{llllll}0,00 & 0,20 & 0,27 & 0,0001 & 2,50\end{array}$

$\begin{array}{llllll}0,00 & 0,20 & 0,27 & 0,0000 & 0,23\end{array}$

$\begin{array}{llllll}0,00 & 0,20 & 0,27 & 0,0000 & 0,31\end{array}$

$\begin{array}{lllll}0,00 & 0,20 & 0,27 & 0,0000 & 1,30\end{array}$

$\begin{array}{llllll}0,00 & 0,20 & 0,27 & 0,0000 & 0,15\end{array}$

$\begin{array}{llllll}0,00 & 0,20 & 0,27 & 0,0000 & 0,20\end{array}$

Quando comparado os resultados obtidos no presente estudo, três anos e meio após o plantio realizado pela prefeitura (SMAC/CRA), observou semelhança quanto à composição florística com 64 espécies $(75,3 \%)$ em comum. Das 85 espécies plantadas, $21(24,7 \%)$ não foram inventariadas no presente estudo (Tabela 3). Em contrapartida, no presente levantamento florístico foram diagnosticadas 25 espécies regenerantes que não constam na lista de plantio, sendo que 4 espécies são consideradas não regionais.

As espécies plantadas e não amostradas no presente estudo, pode simplesmente não ter sido levantadas, nas amostras, ou mais provavelmente que a falta de manutenção acarretou a mortalidade dessas espécies, já que a mortalidade geral foi alta (59\%).

Tabela 3. Relação de espécies que foram plantas em 2011, mas não foram encontradas nas 44 parcelas no Maciço da Tijuca, Rio de Janeiro, RJ, Brasil com as respectivas informações de dispersão, grupo ecológico e demais informações. LEGENDA: D - Dispersão: Ane Anemocórica; Zoo - Zoocórica; Aut - Autocórica; GE - Grupo Ecológico: P - Pioneira; NP - Não Pioneira; E-exótica; NC- Não classificada; Origem: PL - Plantada; RG - Regenerante; ${ }^{* *}$ Espécie não regional.

\begin{tabular}{llcc}
\hline Família & Nome científico & D & GE \\
\hline Fabaceae & Acacia polyphylla DC. & Aut & P
\end{tabular}




\begin{tabular}{lllc} 
Arecaceae & Acrocomia aculeata (Jacq.) Lodd. ex Mart. & Zoo P \\
Meliaceae & Cabralea canjerana (Vell.) Mart. & Zoo P \\
Myrtaceae & Campomanesia xanthocarpa Mart. ex O. Berg & Zoo NP \\
Fabaceae & Centrolobium tomentosum Guillemin ex Benth. & Ane NP \\
Boraginaceae & Cordia superba Cham. & Zoo P \\
Euphorbiaceae & Croton floribundus Spreng. & Aut P \\
Myrtaceae & Eugenea brasiliensis Lam. & Zoo NP \\
Rubiaceae & Genipa americana L. ${ }^{*}$ & Zoo NP \\
Nyctaginaceae & Guapira opposita (Vell.) Reitz & Zoo NP \\
Caricaceae & Jaracatia spinosa (Aubli) A. DC. & Zoo P \\
Fabaceae & Mimosa artemisiana Heringer \& Paula & Ane P \\
Myrtaceae & Psidium myrtoides O. Berg & Zoo NP \\
Fabaceae & Pterogyne nitens Tul. & Ane NP \\
Myrsinacea & Rapanea ferruginea (Ruiz \& Pav.) Mez & Zoo P \\
Fabaceae & Senna macrantera (DC. ex Collad.) H.S. Irwin \& Barneby & Aut P \\
Anacardiaceae & Spondias monbin L. & Zoo NP \\
Fabaceae & Swartzia langsdorffii Raddi & Zoo NP \\
Apocynanceae & Tabernaemontana laeta Mart. & Zoo P \\
Anacardiaceae & Tapirira guianensis Aubl. & Zoo NP \\
Rutaceae & Zanthoxyllum rhoifolium Lam. & Zoo NP \\
\hline
\end{tabular}

O estado do Rio de Janeiro não possui uma lista oficial de espécies ameaçadas ou vulneráveis a extinção. De acordo com decreto municipal 15.793/97 referentes às espécies ameaçadas do município do Rio de Janeiro, das espécies encontradas, estão vulneráveis: Apuleia leocarpa, e Hymenaea coubaril ou em perigo: Brosimum glasiovi, Cariniana ianeirensis e Dalbergia nigra.

Segundo MELO \& DURIGAN (2007), os reflorestamentos têm mantido uma tendência na restauração dos aspectos funcionais, principalmente de matas e conservação da diversidade. Nesse aspecto, ressalta-se que há cinco espécies enquadradas em duas categorias de ameaça, como citado anteriormente, destacando a importância do reflorestamento do Maciço da Tijuca para a conservação dessas espécies e também da biodiversidade. Considera-se que iniciativas de enriquecimento devem ser realizadas com espécies não pioneiras, ou seja, espécies de final de sucessão, em florestas que o dossel já esteja estabelecido, como em algumas áreas amostradas. De modo complementar, sugere-se o uso de espécies que se enquadrem em alguma categoria de ameaça, de forma a promover sua conservação. No entanto, deve-se sempre considerar a proporção em que essas espécies 
aparecem na natureza, evitando-se problema como pragas e doenças, que surgem quando indivíduos de uma espécie são plantados com alta densidade (BARBOSA et al., 2011), ou quando plantados como monoculturas.

Em relação às espécies exóticas encontradas no Maciço da Tijuca, as espécies Bauhinia variegata e Sapindus saponaria foram encontradas plantadas e regenerantes; as espécies Handroanthus impetiginosus, Jacaranda mimosifolia, Lamanonia cuneata, Myracrodruon urundeuva, Samanea saman e Triplaris surinamensis foram encontradas apenas plantadas; já Mangifera indica Musa sp., Persea americana e Syzygium cumini encontradas apenas como regenerantes.

Em relação às espécies exóticas encontradas, algumas delas foram plantadas propositalmente, conforme metodologia de plantio, no entanto, temos que tomar cuidado na escolha das exóticas e sempre que possível optarmos por espécies nativas regionais, já que, o efeito dessas espécies, em ambientes naturais, é hoje a segunda maior causa de extinções no mundo, perdendo apenas para a perda e fragmentação de habitats (BOURSCHEID \& REIS, 2010).

Algumas das exóticas foram plantadas devido a um erro de identificação taxonômica das matrizes fornecedoras de sementes, como a Bauhinia forficata ao invés da exótica Baihinia variegata. Ou ainda essa espécie pode ter sido identificada em nível de gênero no momento.

Já a espécie Psidium guajava (goiabeira) merece especial atenção, pois tem sido amplamente utilizada em reflorestamentos. Essa espécie é considerada subespontânea no Brasil, sendo que as espécies encontradas no país, na maioria dos casos, são cultivares e não a espécie silvestre. A goiabeira é muita atrativa para a fauna, devido à grande quantidade de frutos, podendo ser facilmente dispersa por possuir numerosas sementes. No entanto, o potencial invasor da espécie já foi observado em unidades de conservação (ZVIEJKOVSKI et al., 2009).

Embora a maioria das espécies e dos indivíduos encontrados seja nativa de ocorrência regional, com base em estudos realizados na cidade do Rio de Janeiro, as espécies exóticas merecem especial atenção, pois algumas podem tornar-se invasoras. 
Houve predomínio de espécies pioneiras, no estrato arbóreo com 48,4\% das espécies e 38,9\% são de espécies não pioneiras. A importância de espécies pioneiras, na floresta, está ligada ao estabelecimento de hábitat propício para o desenvolvimento de espécies não pioneiras, que se desenvolvem em ambiente com menor luminosidade. No entanto, o presente estudo foi realizado em uma floresta formada a partir de um reflorestamento, em que indivíduos pioneiros e não pioneiros foram plantados simultaneamente, havendo crescimento simultâneo de espécies desses dois grupos sucessionais. Nesta situação, segundo WHITMORE (1989), as espécies pioneiras possuem crescimento mais rápido do que as espécies não pioneiras, ocupando o dossel da floresta, sendo posteriormente substituídas por não pioneiras.

Outro fator importante, em uma área em processo de restauração, é a incorporação de biomassa, que pode ser avaliada em função da área basal, expressa pela Dominância Relativa (DoR), obtida a partir da análise fitossociológica (tabela 2). Entre as espécies que apresentaram maior DoR, são: Gochnatia polymorpha, Machaerium hirtum, Alchornea triplinervia, Sparattosperma leucanthum, Pseudobombax grandiflorum e Guarea guidonia. Exceto Machaerium hirtum e Pseudobombax grandiflorum as outras são pioneiras e representam juntas $62,2 \%$ da dominância relativa. As espécies pioneiras, de forma geral, contribuíram em $83,4 \%$ na área basal, enquanto as espécies não pioneiras contribuíram em $16,6 \%$ do total das espécies amostradas.

A importância de indivíduos e espécies pioneiros também está relacionada à contribuição significativa para o acúmulo de biomassa e, consequentemente, no acúmulo de matéria orgânica no ecossistema.

A área basal, de $4,17 \mathrm{~m}^{2} /$ ha não foi tão significativa, comparado a outros trabalhos com idade semelhantes. DAMASCENO (2005) também observou um aumento de área basal de $15,19 \mathrm{~m}^{2} / \mathrm{ha}$, aos seis anos. MANDETTA (2007) achou $9,27 \mathrm{~m}^{2} /$ ha em um reflorestamento de 2,5 anos.

Por outro lado, a densidade do plantio usada em cada reflorestamento pode interferir no cálculo da área basal, muito embora MELO \& DURIGAN (2007) também considerem a área basal como o melhor indicador para se avaliar uma comunidade e para comparar diferentes comunidades. 
A altura da vegetação do Maciço da Tijuca, também não foi satisfatória, já que $90 \%$ dos indivíduos, plantados ou regenerantes, tiveram até $3 \mathrm{~m}$ de altura. A altura predominantemente ficou entre 0,1 e $1 \mathrm{~m}$ para $81 \%$ dos indivíduos plantados e até $3 \mathrm{~m}$ para $87,5 \%$ dos indivíduos regenerantes. Árvores emergentes foram Alchornea triplinervia para espécies regenerantes, que chegou a $12 \mathrm{~m}$, já para as espécies plantadas Tibouchina sellowiana (9m) e Peltophorum dubium (6m).

Em um estudo realizado em floresta ombrófila densa, ARZZOLA (2011) também observou que, em uma área de regeneração natural, Croton floribundus foi a espécie com maior crescimento, ultrapassando os 18 metros de altura, aproximadamente quatro anos após o corte raso. Esse fato ocorreu devido ao rápido crescimento das espécies pioneiras, em relação às demais, formando, predominantemente, o dossel da floresta.

De acordo com SOUZA \& BATISTA (2004), a altura pode ser um bom descritor para a vegetação, desde que ainda esteja em desenvolvimento inicial. Os autores observaram que em reflorestamentos que possuem cerca de cinco anos, há um favorecimento da altura, em detrimento da área basal. Esse fato ocorre devido ao crescimento de espécies pioneiras, que proporcionam cobertura do solo e fechamento da copa. No entanto, os mesmos autores observaram que reflorestamentos, a partir de 10 anos, apresentam diferença de área basal em relação a reflorestamentos mais recentes, mostrando a importância desse parâmetro para florestas dessa idade.

Em relação à densidade de indivíduos, observa-se elevado número encontrado no presente estudo. Esse fato pode estar associado metodologia de amostragem, já que no presente estudo todos os indivíduos foram amostrados, independente da altura e diâmetro dos indivíduos. Por outro lado a presente metodologia pode trazer indícios das condições do componente regenerante da área, de forma complementar aos dados do plantio.

Vários aspectos interferem no processo de colonização de uma área em processo de sucessão, como presença de fragmentos próximos, banco de sementes, histórico do uso da área, entre outros (GUARIGUATA \& OSTERTAG, 2001; RODRIGUES et al., 2011).

Destaca-se ainda que, após o fechamento da copa nas florestas secundárias, as taxas de ciclagem de nutrientes e a produtividade tendem a ser 
altas (GUARIGUATA \& OSTERTAG, 2001). SALDARRIAGA et al. (1988) observaram que entre 60 e 80 anos após corte e queima, espécies típicas de florestas maduras começam a ocorrer em florestas secundárias e estimaram em torno de 190 anos, para que uma floresta secundária recupere a área basal e biomassa de uma floresta madura. Observa-se ainda que, em florestas maduras, as espécies tardias são mais abundantes e apresentam maior área basal do que em florestas secundárias. (CHAZDON et al., 2010).

Em relação à síndrome de dispersão, nota-se que as espécies zoocóricas foram predominantes quanto à proporção de espécies. No entanto, não observa-se predomínio em relação ao número de indivíduos para essa síndrome. As espécies zoocóricas têm contribuído para a atração da fauna no Maciço da Tijuca.

As espécies zoocóricas são de fundamental importância, no Maciço da Tijuca, pois atraem a fauna, favorecendo a chuva de sementes e, consequentemente, contribuindo com o enriquecimento da área com novas formas de vida, o que pode facilitar a restauração (MELO \& DURIGAN, 2007). No entanto, destaca-se que no estrato arbóreo ainda não há reflexo de forte influência de novas espécies, permitindo destacar que sua composição florística ainda está sujeita, predominantemente, ao que se plantou.

Destaca-se também a importância das espécies anemocóricas, que atuam como fonte de propágulos autóctones, e como fornecedoras de sementes para fragmentos próximos. As espécies anemocóricas foram influenciadas principalmente pela densidade de indivíduos pertencentes às espécies da família Bignoniaceae.

Em relação à presença das espécies autocóricas, expressiva no estrato arbóreo, representam mais que $20 \%$ das espécies, sendo influenciadas principalmente por espécies pertencentes à família Fabaceae.

Com base nos resultados obtidos a partir dos índices de Shannon, observa-se uma relativa diversidade do Maciço da Tijuca, sendo pouco abaixo a outros reflorestamentos realizados no estado do Rio de Janeiro, com idades semelhantes ou superiores (MULLER, 2014; MOURA et al., 2011).

No entanto, observa-se que não é suficiente apenas reflorestar uma área com alta riqueza de espécies, mas também é necessário haver 
equitatividade na distribuição dos indivíduos, semelhante a áreas naturais do Estado do Rio de Janeiro, conforme verificado por DURIGAN \& LEITÃO-FILHO (1995).

TABARELLI \& MANTOVANI (1999) observaram que, nas áreas em processo de sucessão, a riqueza e a diversidade são características que se recuperam antes de outros fatores, como a composição de guildas, composição florística estrutura física, com exceção da densidade.

Esse modelo também é aplicável a áreas que estão em processo de restauração. No entanto, observa-se que a riqueza e diversidade são definidas no momento do plantio, podendo sofrer influências externas ao longo do tempo. Alguns fatores que exercem essas influências referem-se ao histórico de uso da área, à proximidade de remanescentes florestais naturais, à ação de animais dispersores no reflorestamento e à manutenção adotada para a área. No entanto, quando não há influência desses fatores, a diversidade do reflorestamento fica restrita ao que se plantou e, nesse contexto, é muito importante utilizar a alta diversidade nos plantios, de forma a proporcionar sustentabilidade ao reflorestamento ao longo do tempo (RODRIGUES et al. 2011).

Parâmetros como a área basal, altura média e diversidade são bons descritores e podem ser utilizados como indicadores para o estágio e a qualidade da vegetação arbórea. Com base nesses parâmetros, observa-se que o reflorestamento do Maciço da Tijuca não apresenta um estrato arbóreo bem desenvolvido, fato talvez explicado pela falta de manutenção e idade do reflorestamento, que afetou negativamente a estrutura do reflorestamento do Maciço da Tijuca.

Observa-se que a área estudada ainda é recente, com apenas 3,5 anos, e seu estrato arbóreo está majoritariamente caracterizado pela composição de espécies utilizadas no plantio. Em longo prazo, espera-se que parâmetros como riqueza e diversidade possam variar, em função dos fatores externos e da própria dinâmica da floresta, principalmente do estrato regenerantes constatado no presente trabalho. 


\section{REFERÊNCIAS BIBLIOGRÁFICAS}

APG III. An update of the Angiosperm Phylogeny Group classification for the orders and families of flowering plants: APG III. Botanical Journal of the Linnean Society 161: 105-121. 2009.

ARZZOLA, F.A.R.P. Florestas secundárias e a regeneração de natural de clareias antrópicas na serra da Cantareira, SP. 2011. Tese (Doutorado em Biologia) - Universidade Estadual de Campinas, Campinas.

BARBOSA, L.M., BARBOSA, T.C., BARBOSA, K.C., PARAJARA, F.C. Práticas e políticas públicas para a restauração ecológica a partir de reflorestamentos com alta diversidade de espécies regionais. In: S.V. Martins (ed.). Restauração ecológica de ecossistemas degradados. Editora UFV, Viçosa, 2012. pp. 240261.

BOURSCHEID, K., REIS, A., Dinâmica da invasão de Pinus elliotti Engelm. Em restinga sob processo de restauração ambiental no Parque Florestal do Rio Vermelho, Florianópolis, SC. Biotemas, 23: 23 - 30. 2010.

BRANCALION, P.H.S., VIANI, R.A.G., RODRIGUES, R.R.R., GANDOLFI, S. Avaliação e monitoramento de áreas em processo de restauração. In: S.V. Martins (ed.). Restauração ecológica de ecossistemas degradados. Editora UFV, Viçosa, 2012. pp. 262-293.

CHAZDON, R.L., FINEGAN, B., CAPERS, R.S., SALGADO- NEGRET, B., CASANOVES, F., BOUKILI, V., NORDEN, N. Composition and dynamics of functional groups of trees during tropical forest succession in Northeastern Costa Rica. Biotropica 42: 31-40. 2010.

COLMANETTI, M.A.A. Estrutura da vegetação e características edáficas de um reflorestamento com espécies nativas. 2013. Dissertação (Mestrado Biodiversidade Vegetal e Meio Ambiente) - Instituto de Botânica, São Paulo.

DAMASCENO J, G. A., SEMIR, J. SANTOS, F. A. M., LEITÃO-FILHO, H. F. Structure, distribuition of species and inundation in riparian Forest of Rio Paraguai, Pantanal, Brazil. Flora, 200:119-135. 2005.

DEAN, W. A ferro e fogo: a história e a devastação da Mata Atlântica brasileira. 6 ed. São Paulo: Companhia das letras, 2007.

DURIGAN, G., LEITÃO-FILHO, H.F. Florística e fitossociologia de matas ciliares do oeste paulista. Revista do Instituto Florestal 7: 197-239. 1995. 
GUARIGUATA, M.R., OSTERTAG, R. Neotropical secondary forest succession: changes in structural and functional characteristics. Forest Ecology and Management 148: 185-206. 2001.

KÖPEN, W. Climatologia. México: Fondo de Cultura económica. 1948.

MANDETTA, E.C.N. Avaliação florística e de aspectos da estrutura da comunidade de um reflorestamento com dois anos e meio de implantação no município de Mogi - Guaçu/SP. 2007. Dissertação (Mestre em Ciências Biológicas) - Universidade Estadual Paulista, Rio Claro.

MARTINS, S.V., RODRIGUES, R.R., GANDOLFI, S., CALEGARI, L. Sucessão Ecológica: Fundamentos e aplicações na restauração de ecossistemas florestais. In: S.V. Martins (ed.). Ecologia de florestas tropicais do Brasil. 2 ed. Editora UFV, Viçosa, 2012. pp. 21-52.

MELO, A.C.G. \& Durigan, G. Evolução estrutural de reflorestamentos de restauração de matas ciliares no Médio Vale do Paranapanema. Scientia Florestalis 73: 101-111. 2007.

MISSOURI BOTANICAL GARDEN'S VAST (Vascular Tropicos) nomenclatural database - W3 Tropicos. http://www.tropicos.org. Acessado em 01 de Junho de 2015).

MOURA, C. J. R., ROCHA, C. S., FONTES, C. V. A., SOUZA, D. M. S., SOUZA, L. G. E., SILVA, P. S., EBISAWA, S. M., NEVES, V. C. Projeto de monitoramento de reflorestamento, "Mutirão Reflorestamento, prefeitura Rio de Janeiro". Rio de Janeiro, RJ. 2011.

MUELLER-DOMBOIS, D., ELLENBERG, H. Aims and methods of vegetation ecology. John Wiley, New York. 1974.

MULLER, A. E. Avaliação de uma Floresta Atlântica urbana em restauração: da ecologia às questões sociais. 2014. Dissertação (Mestrado em Botânica Tropical) - Instituto de Pesquisas Jardim Botânico do Rio de Janeiro, RJ.

MYERS, N., MitTERMEIER, R. A., MITTERMEIER, C. G., DA FONSECA, G. A. B. \& KENT, J. Biodiversity hotspots for conservation priorities. Nature 403: 853-858. 2000.

PIELOU, E.C. An introduction to mathematical ecology. Wiley, New York. 1969. RIO DE JANERIO. Resolução INEA № 36, de 08 de julho de 2011. Aprova o Termo de Referência para Elaboração de Projetos de Recuperação de Áreas Degradadas - PRAD. Rio de Janeiro, 1ํ de Julho de 2011. 
RIO DE JANERIO. Resolução INEA № 89, de 03 de junho de 2014. Dispõe sobre as proporções mínimas aplicáveis para reposição florestal, decorrentes do corte ou supressão de vegetação pertencente às formações florestais nativas e ecossistemas associados do bioma Mata Atlântica, bem como de intervenções em áreas de preservação permanente APP, para fins de licenciamento ambiental e/ou de autorização para supressão de vegetação nativa - ASV no estado do Rio de Janeiro. 1ํ de Junho de 2014.

RODRIGUES, R.R., GANDOLfi, S., NAVE, A.G.; ARONSON, J., BARRETO, T.E., Vidal C.Y., BRANCALION, P.H.S. Large-scale ecological restoration of high-diversity tropical forests in SE Brazil. Forest Ecology and Management 261: 1605-1613. 2011.

SÃO PAULO. 2008. Resolução SMA n ${ }^{\circ} 8$, de 31 de janeiro de 2008. Diário Oficial do Estado de São Paulo - Meio Ambiente, 01/02, pp. 31-32.

SALDARRIAGA, J.G., WEST, D.C., THARP, M.L., UHL, C. Long-term chronosequence of forest succession in the upper Rio Negro of Colombia and Venezuela. Journal of Ecology 76: 938-958. 1988.

SOS MATA ATLÂNTICA. Atlas dos remanescentes florestais da Mata Atlântica, período de 2008 a 2010. Instituto Nacional de Pesquisas Espaciais. 2010. Disponível em <http:// www.sosmatatlantica.org.br>. Acessado em: 15 de Dezembro de 2014.

SOUZA, F.M., BATISTA, J.L.F. Restoration of seasonal semideciduous forests in Brazil: influence of age and restoration design on forest structure. Forest Ecology and Management 191: 185-200. 2004.

TABARELLI, M., MANTOVANI, W. A regeneração de uma floresta tropical montana após corte e queima (São Paulo - Brasil). Revista Brasileira de Biologia 59: 239-250. 1999.

WHITMORE, T. C. Canopy gaps and the two major groups of forest trees. Ecology 70: 536-538. 1989.

ZVIEJKOVSKI, I.P., CAMPOS, J.B., CAMPOS, R.M. \& LANDGRAF, G.O. Potencial invasor de Psidium guajava. Em um intervalo de cinco anos (2002 2007) dentro de uma Unidade de Conservacão. In: W. Delitti (org.). Anais do IX Congresso de Ecologia do Brasil, São Lourenço, 2009.

Recebido: 08/06/2015

Aprovado: 29/06/2015 